\title{
AlGaAs/GaAs Heterojunction Bipolar Transistors on Si Substrate Using Epitaxial Lift-Off
}

\author{
J. C. Fan, C. P. Lee, Member, IEEE, J. A. Hwang, and J. H. Hwang
}

\begin{abstract}
Epitaxial lift-off (ELO) technique was used for the first time to transplant AlGaAs/GaAs Heterojunction Bipolar Transistors (HBT's) to Si substrates. Both preprocessed (devices processed before transplantation) and postprocessed (devices processed after transplantation) ELO HBT's on Si were demonstrated in this study. The characteristics of those HBT's on Si with either technique show nearly identical characteristics of the HBT's on GaAs without transplantation, indicating the film quality is maintained after transplantation. Devices with a high current gain of 550 were transplanted without any degradation, and the current gain is not limited by the ELO process. This current gain value is the highest reported for GaAs HBT's on $\mathrm{Si}$ with any techniques.
\end{abstract}

\section{INTRODUCTION}

I

NTEGRATING semiconductor devices with different func-

tions, structures and materials on a same substrate has always been attractive for many device applications. This is particularly true for optoelectronic devices where optical devices and electronic devices have to work together. Although optoelectronic integrated circuits (OEIC's) have many advantages compared with hybrid circuits, the incompatibility of structures and materials between devices makes the integration very difficult. An alternative to direct integration is to use wafer bonding techniques [1]-[3] where two different wafers are physically bonded together. Of the various techniques developed, the epitaxial lift-off (ELO) technique developed by Yablanovitch et al. [1] is particularly useful for the fabrication of integrated III-V semiconductor devices. In this technique, a very thin epitaxial layer is lifted off from its host substrate and bonded to another substrate by van der Waal's force. Successful transfer of GaAs MESFET's to a Si substrate has already been demonstrated [4]. In this letter, we report, for the first time, transfer of GaAs/AlGaAs heterojunction bipolar transistors (HBT's) to a Si substrate using the ELO technique. HBT's have been shown in recent years as one of the most promising devices for high speed applications. Transplanting HBT circuits to Si wafers makes it possible to combine the high speed capability of HBT front end with the processing power of Si VLSI's. Besides, the high thermal conductivity of Si can help solve the power dissipation problem of high performance HBT circuits. HBT's, different from MESFET's, are minority carrier devices and are vulnerable to damages

Manuscript received January 19, 1995; revised April 28, 1995. This work was supported by the National Science Council under Contract NSC84-2215E009-033.

The authors are with the Department of Electronics Engineering and Institute of Electronics, National Chiao Tung University, Hsinchu 300, Taiwan, R.O.C.

IEEE Log Number 9413807.

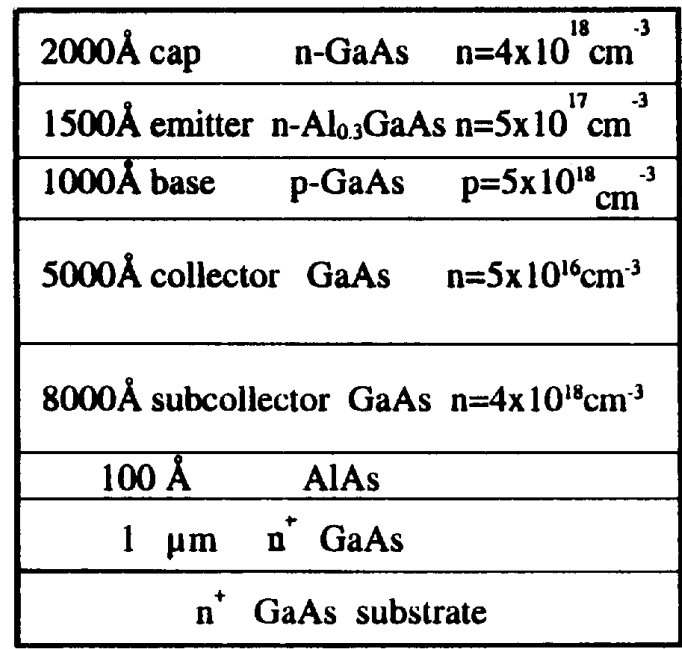

Fig. 1. The layer structure of HBT's used in this study. The $100 \AA$ AlAs layer serves as a release layer during the ELO process.

which may be incurred during the transplantation process. In the past, the integration of HBT's with $\mathrm{Si}$ devices has been attempted by epitaxial growth of HBT structures directly on Si substrates [5], [6]. But, because of the large lattice mismatch between the two materials, the resulting dislocations and lattice defects limit the performance of the HBTs. The highest current gain obtained is around 100 for a current density of $1 \times 10^{4} \mathrm{~A} / \mathrm{cm}^{2}$ [5]. The ELO process described below, however, shows it is capable of maintaining the performance of the HBTs without any degradation. Since the lifted film is very thin, we are able to fabricate devices either before or after transplantation (preprocessed or postprocessed ELO technique) and obtain similar results.

\section{EXPERIMENTAL RESULTS AND DISCUSSION}

The HBT samples used for this study were grown on (100) $N^{+}$GaAs substrates by a Varian Gen II molecular beam epitaxy (MBE) system. The layer structure is shown in Fig. 1. It is a standard NPN HBT with an abrupt emitter-base junction. The $P^{+}$GaAs base has a thickness of $1000 \AA$ and a doping concentration of $5 \times 10^{18} \mathrm{~cm}^{-3}$. Below the subcollector, a 100 $\AA$ AlAs layer was grown. This layer is used as a release layer which is to be etched during the lift-off process. Two samples were prepared. For the postprocessed sample (sample A), we lifted the HBT structure and grafted it onto the Si substrate before we did the device processing. For the preprocessed 


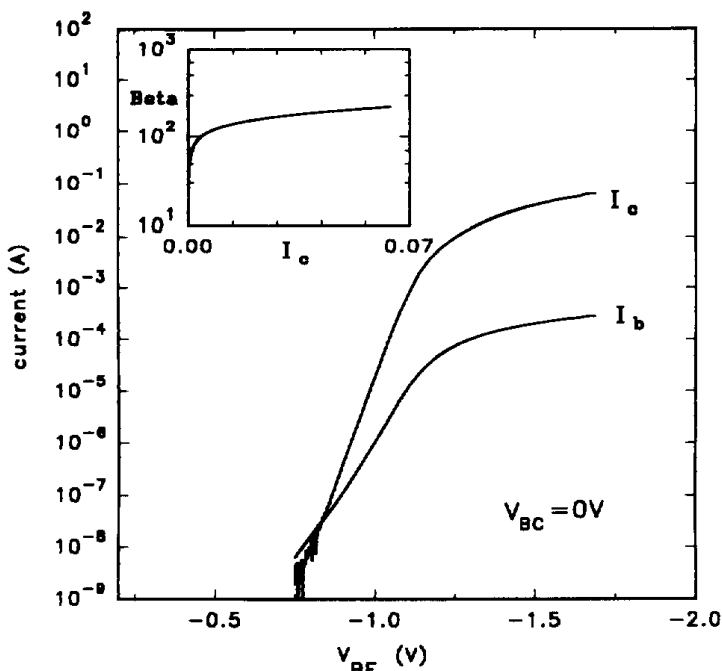

(a)

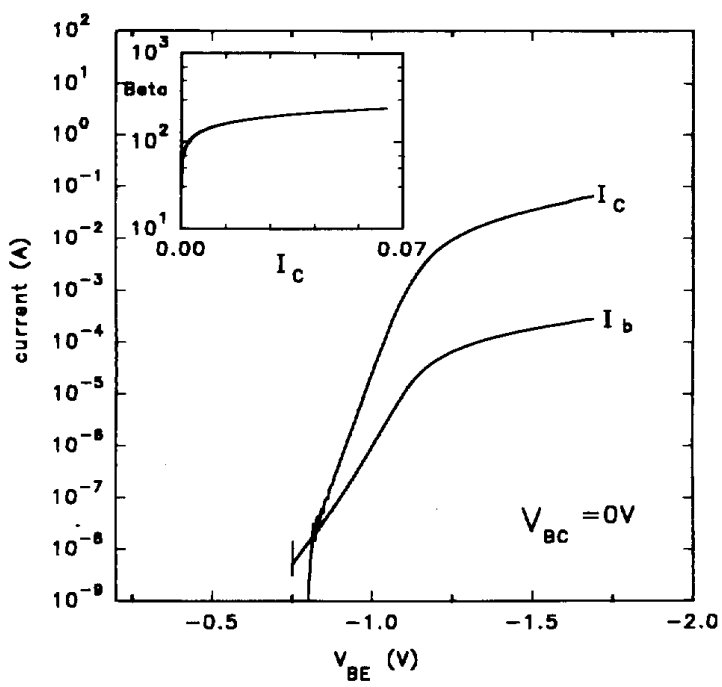

(b)

Fig. 2. The Gummel plots and the current gain versus collector current plots of (a) an HBT fabricated on a GaAs substrate and (b) an HBT fabricated on the transplanted film on a $\mathrm{Si}$ substrate.

sample (sample B), we processed the HBT's first and then grafted the HBT's onto the Si substrate. The lift-off process is the same for both cases. Apiezon $\mathrm{W}$ wax was first applied to the specimen and then the sample was placed in a $10 \%$ HF solution overnight to selectively etch away the $100 \AA$ AlAs release layer. After the semiconductor film was lifted off from the GaAs substrate, it was rinsed in D.I. water and then transferred onto the $\mathrm{Si}$ substrate. The most crucial step in the ELO process is the transferring of the epilayer to the Si substrate. The surface of the Si substrate has to be smooth and free from any foreign particles. Extreme care has to be taken when placing the epilayer onto the Si wafer to prevent any foreign material being trapped at the interface which may cause bilistering in the film during subsequent processing and eventually device failure. The HBT process follows the

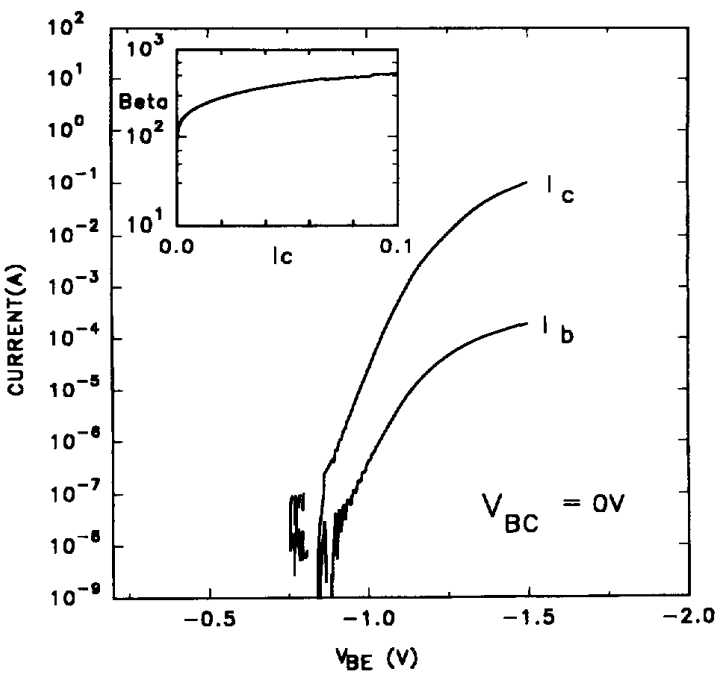

(a)

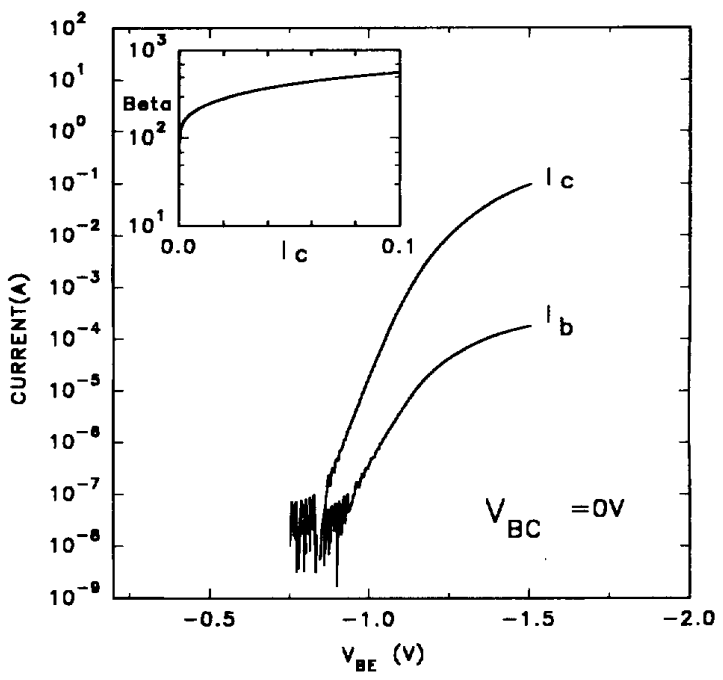

(b)

Fig. 3. The Gummel plots and the current gain versus collector current plots of a preprocessed HBT (a) on a GaAs substrate and (b) after transplantation to a $\mathrm{Si}$ substrate.

standard procedure including photolithography, mesa etching, and ohmic contact metallization. The emitter dimension was $50 \mu \mathrm{m} \times 50 \mu \mathrm{m}$.

To compare the performance of the HBT's before and after the transplantation, we also processed HBT's directly on GaAs substrates without transplantation for both sample $A$ and sample B. Fig. 2(a) and (b) shows the Gummel plots for an HBT-on-GaAs and a postprocessed ELO HBT on $\mathrm{Si}$, respectively. The current gain as a function of the collector current (at $V_{b c}=0 \mathrm{~V}$ ) is shown in the inset of each figure. The devices have nearly identical characteristics. The HBT fabricated on a grafted film did not show any performance degradation compared with that fabricated on a GaAs substrate. They both have a maximum current gain around 200 . The ideality factors for the collector current and the base current were 1.08 and 
1.5, respectively, for both devices. So from this comparison, we demonstrate that the film quality is maintained after the liftoff process. Since the HBT's were processed after the epilayers were grafted onto the $\mathrm{Si}$ wafer, the result shown here also demonstrates that the van der Waal's force, which is responsible for the bonding between the epilayer and the Si substrate, is strong enough to hold the film through device processing.

For sample B, we processed HBT's before lift-off. In order to show that the ELO process does not cause any degradation for even very small base current, the HBT's were processed with an emitter ledge passivation technology. The emitter ledge on the extrinsic base surface had a thickness of 500 $\AA$ to ensure full depletion. After device fabrication, part of the sample underwent the ELO process and the film with the completed HBT's was grafted onto a Si substrate. We then compared the characteristics of devices after transplantation with those which were not transplanted. Fig. 3(a) and (b) shows the Gummel plots and the current gain versus collector current plots for a preprocessed ELO HBT on Si and a HBT without transplantation, respectively. Because of the emitter ledge, the base currents are lower and the gains are higher compared with those shown in Fig. 2. Again, no obvious difference in the characteristics was observed in these two devices. The current gain was around 550 (at a collector current of $0.1 \mathrm{~A}$ ) for both devices. The ideality factors were 1.17 and 1.43 for the collector current and the base current, respectively. So even though the ELO process is done after the devices are completed, the lift-off process does not cause any device degradation. An interesting application of this technique is to place and align the GaAs thin film on specified locations of $\mathrm{Si}$ integrated circuits. Although not demonstrated in this experiment, this is certainly possible and has been demonstrated in [7]

\section{CONCLUSION}

We have used the epitaxial lift-off technique to put AlGaAs/GaAs HBT's on Si substrates. Both preprocessed and postprocessed ELO HBT's have been fabricated. The device characteristics measured on HBT's with and without transplantation show no degradation for both preprocessed sample and the postprocessed sample. Because the lifted-off film is very thin in this technique, device process can be done either before or after transplantation. In the preprocessed sample, we have successfully transplanted an HBT with a current gain of 550 to a $\mathrm{Si}$ substrate. The value is larger than the highest reported current gain value $(\sim 100)$ for AlGaAs/GaAs HBT's grown directly on Si. It should be mentioned that the current gain is not limited by the ELO process. This study demonstrates the feasibility of quasi-monolithically integrating high-speed AlGaAs/GaAs HBT devices with high density silicon VLSI circuits. The high heat conductivity of Si substrate (compared to that of $\mathrm{GaAs}$ ) is also helpful to alleviate the heat dissipation problem for high power GaAs devices.

\section{REFERENCES}

[1] E. Yablonovitch, T Gmitter, J. P. Harbison, and R. Bhat, "Extreme selectivity in the lift-off of epitaxial GaAs films," Appl. Phys. Lett., vol. 51, pp. 2222-2224, 1987.

[2] I. Pollentier et al., "Epitaxial lift-off GaAs LEDs to Si for fabrication of opto-electronic integrated circuits," Electron. Lett., vol. 26, pp. 193-194, 1990.

[3] Y. H. Lo, R. Bhat, D. M. Hwang, M. A. Koza, and T. P. Lee, "Bonding by atomic rearrangement of InP/nGaAsP $1.5 \mu \mathrm{m}$ wavelength lasers on GaAs substrates," Appl. Phys. Lett., vol. 58, pp. 472-473, 1991.

[4] I. Pollentier, C. Brys, P. Demeester, P. Van Daele, and L. Martens, "Transplantation of epitaxially lifted-off MESFETs fabricated by a commercial foundry," Electron. Lett., vol. 29, pp. 291-293, 1990.

[5] W. Liu, and S.-D. Kim, "A high-gain AlGaAs/GaAs heterojunction bipolar transistor grown on silicon substrates," Jpn. J. Appl. Phys., vol. 31, pp. 2656-2659, 1992.

[6] T. Won, C. W. Litton, H. Morkoc and A. Yariv, "A high-gain GaAs/AlGaAs n-p-n heterojunction bipolar transistor on (100) Si grown by molecular beam epitaxy," IEEE Electron Device Lett., vol. 9, pp. 405-407, 1988.

[7] C. Camperi-Ginestet, M. Hargis, N. Jokerst, and M. Allen, "Alignable epitaxial liftoff of GaAs materials with selective deposition using polyimide diaphragms," IEEE Trans. Electron Devices, vol. 3, pp. 1123-1126, 1991. 